\title{
Application of the drag force method to evaluate magnetic property degradation near the cut edges of electrical steels
}

\author{
Ivan J. Garshelis, ${ }^{1,2, a)}$ Guillaume Crevecoeur, ${ }^{3}$ Stijn P. L. Tollens ${ }^{2}$ and Luc Dupre ${ }^{3}$ \\ ${ }^{1}$ Magnova, Inc., Pittsfield, Massachusetts 01201, USA \\ ${ }^{2}$ MagCanica, Inc., San Diego, California 92117, USA \\ ${ }^{3}$ Department Electrical Energy, Systems and Automation (EESA), Ghent University, B-9000 Ghent, Belgium
}

(Presented 18 November 2010; received 1 October 2010; accepted 30 November 2010; published online 1 April 2011)

\begin{abstract}
The increase in hysteresis loss associated with the altered microstructure and residual stress fields in regions near the cut edges of electrical steels is investigated by means of drag force measurements. Measurements are made using relatively narrow magnets on samples of two grades of nonoriented steels cut by laser or mechanical processes. Largest drag forces, hence losses, are consistently found in slow laser cut samples, smallest drag forces with fast laser cut samples, and moderately higher losses in mechanically cut samples. These results are consistent with other measurement methods. (ㅇ 2011 American Institute of Physics. [doi:10.1063/1.3556943]
\end{abstract}

\section{INTRODUCTION}

By the unavoidable introduction of microstructural changes and residual stresses, the cutting of steel sheets to the size and shape required to produce laminated magnetic cores degrades the key magnetic properties in the material near the cut edges. Thus, permeability is decreased, and hysteresis loss is increased in a zone varying from 1 to 5 times the sheet thickness and depending on the cutting process. ${ }^{1,2}$ Previous observation ${ }^{3}$ of these degradations has employed the needle probe method wherein the voltage between needles placed on the surface of a sample subjected to a low frequency alternating field is measured. This methodology contains, however, approximations ${ }^{4}$ and is exhaustive.

The drag force method (DFM) involves the measurement of the lateral forces, $F$, acting between a permanent magnet (PM) and a proximate ferromagnetic strip as the strip is moved first in one direction and then in reverse. ${ }^{5}$ The algebraic difference in the forces measured during motion in each direction, i.e., $\Delta F$, has been shown to be a measure of the hysteresis loss associated with the induction extremes provided by the PM. In contrast to previous applications of the DFM wherein the magnets were wide enough to magnetize the full strip width, the magnets employed in this study are both significantly narrower and are positioned at or near the edge being examined. The measured $\Delta F$ values, being related to the hysteresis losses in bands commensurate with the PM width, are found to reflect the relative severity of the degradation due to the different cutting processes.

\section{THEORY}

That the DFM can reveal both the relative intensity and spatial extent of the degraded regions is based on the following simplified analysis. Reference is made to Figs. 1 and 2, which shows the physical arrangement of a PM and the and

\footnotetext{
${ }^{a)}$ Electronic mail: ijgarsh@att.net.
}

the strip sample under test (SUT), and identifies the significant parameters.

Starting with the assumption that, within a thin sample $\left(t_{\mathrm{S}}\right.$ in Fig. 1$)$, a longitudinally magnetized band of material having a width equal to or commensurate with the width of the magnet $\left(W_{\mathrm{M}}\right.$ in Fig. 1) is created during the indicated motion of the sample. Within this band, the magnetization, $M_{x}$, will vary with distance, $x$, from the PM, and for a PM close enough to the sample ( $G$ in Fig. 1) and having a large enough moment, $m$, this distribution will include local regions having a full range of magnetizations from near positive saturation $(x=0)$ to near negative saturation $\left(x= \pm 1.225 G_{\mathrm{e}}\right)$ (Ref. 5) and all values between these two extrema. For any value of $x, M_{x}$ will depend on the longitudinal component of the field $H_{x}$ from the PM and on the history of exposure to this field (and those associated with the $\nabla \cdot M_{x}$. It has been shown ${ }^{5}$ that mutually repulsive longitudinal forces equal to $\int M_{x} \operatorname{grad} H_{x} \mathrm{~d} x$ act between the magnetized material on either side of the PM and the PM. Although $H_{x}=H_{-x}$, following from the hysteretic relationship between $M$ and $H$ in the sample material, $M_{x} \neq M_{-x}$, hence the forces acting in each direction are not equal, hence a net force, i.e., the drag force, acts to pull the magnet in the direction of the

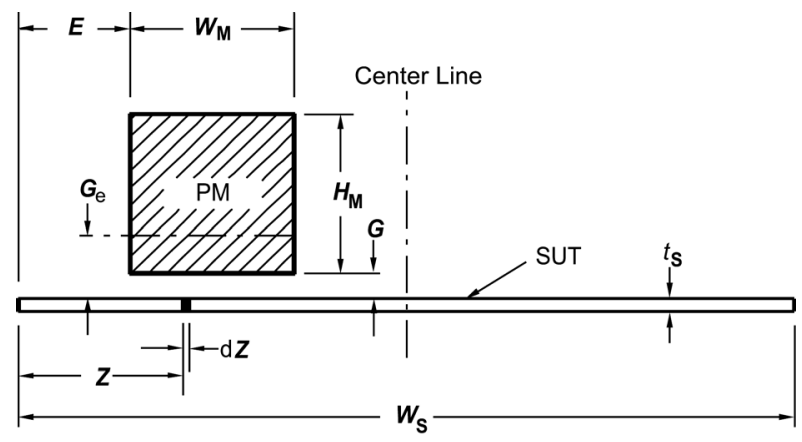

FIG. 1. Physical arrangement of magnet and sample together with defining parameters. $G_{\mathrm{e}}$ is the effective gap, i.e., the location of a linear array of dipoles having the combined moment of the PM and the same field intensity at the SUT surface. 


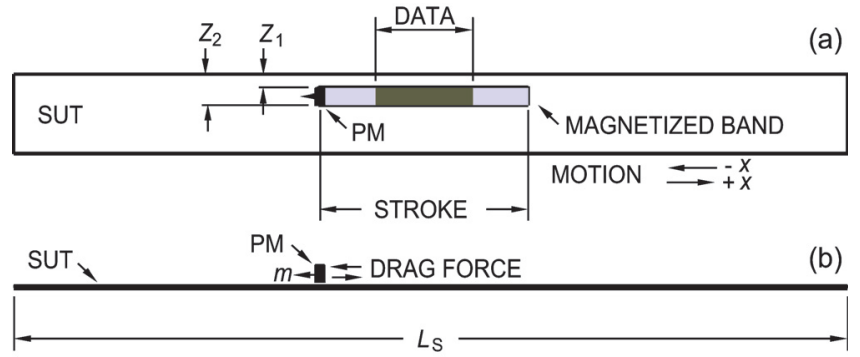

FIG. 2. (Color online) (a) Top view of elements shown in Fig. 1. STROKE defines the range of the back and forth motion of the SUT. (b) Side view: $m$ indicates the magnetic moment of the PM.

SUT motion. Since $\mathrm{d} H / \mathrm{d} x$ becomes very $\operatorname{small}^{5}$ for $x$ $\geq\left| \pm 5 G_{\mathrm{e}}\right|$, the magnitude of this force, $F$, is found closely as

$$
F \approx A \int_{-5 G_{\mathrm{e}}}^{+5 G_{\mathrm{e}}} M_{x}\left(\frac{\mathrm{d} H}{\mathrm{~d} x}\right)_{x} \mathrm{~d} x,
$$

where $A=W_{\mathrm{M}} t_{\mathrm{s}}$ is the cross sectional area of the magnetized region, and the integral is seen to be the sum of the hysteresis loss densities associated with traversal of a major loop together with traversal of a "minor" loop having extrema at saturation and remanence.

Since the local hysteresis loss will expectedly vary, from a maximum at or near the edge and diminish with increasing distance from the edge, the contribution to $F$ from each elemental width ( $\mathrm{d} z$ in Fig. 1) will expectedly vary with $z$. The measured drag force for a band located as indicated in Fig. 2(a) will then be found as

$$
F \approx t_{\mathrm{s}} \int_{z_{1}}^{z_{2}} \int_{-5 G_{\mathrm{e}}}^{+5 G_{\mathrm{e}}} M_{x, z}\left(\frac{\mathrm{d} H}{\mathrm{~d} x}\right)_{x} \mathrm{~d} x \mathrm{~d} z .
$$

For a band wherein $\mathrm{z}_{1}=E$ (in Fig. 2) and $z_{2}-z_{1}=W_{\mathrm{M}}$, the measured drag force would expectedly be highest when $E=0$ and decrease with the increasing $E$ toward the value measured at the center of the SUT over a range of $E$ reflective of the extent of the affected zone.

\section{EXPERIMENT AND DISCUSSION}

Fully processed nonoriented steel sheets $0.5 \mathrm{~mm}$ thick, of grades $350-50 \mathrm{~A}(\mathrm{Si} \approx 2 \%)$ and $800-50 \mathrm{~A}(\mathrm{Si} \approx 1.3 \%)$, were cut into $30 \mathrm{~mm}$ wide, $300 \mathrm{~mm}$ long strips. "A" samples were cut at $100 \mathrm{~mm} / \mathrm{s}$ by a continuous $\mathrm{CO}_{2}$ laser $(1 \mathrm{~kW}$ power and coaxial oxygen as assisting gas). "B" samples were similarly cut at $20 \mathrm{~mm} / \mathrm{s}$. "C" samples were mechanically sheared.

TABLE I. $\Delta F$ for PMs and SUTs indicated (350 grade).

\begin{tabular}{lccccc}
\hline \hline $\begin{array}{l}\text { PM } \Rightarrow \\
\text { SUT } \Downarrow\end{array}$ & $\begin{array}{c}6.35 \mathrm{~mm} \\
\text { Edge }(\mathrm{mN})\end{array}$ & $\begin{array}{c}12.7 \mathrm{~mm} \\
\text { Edge }(\mathrm{mN})\end{array}$ & $\begin{array}{c}12.7 \mathrm{~mm} \\
\text { Center }(\mathrm{mN})\end{array}$ & $\begin{array}{c}50.8 \mathrm{~mm} \\
\text { Center }(\mathrm{mN})\end{array}$ & $\begin{array}{c}\text { Hysteresis loss } \\
\left(\mathrm{J} / \mathrm{m}^{3}\right)\end{array}$ \\
\hline A1 & 3.39 & 7.68 & 7.68 & 11.13 & 379 \\
$\mathrm{~A} 2$ & 3.17 & 7.75 & 7.08 & 10.48 & 355 \\
$\mathrm{~B} 1$ & 4.26 & 9.78 & 7.63 & 12.06 & 401 \\
$\mathrm{~B} 2$ & 4.27 & 9.94 & 8.60 & 11.51 & 384 \\
$\mathrm{C} 1$ & 3.70 & 7.80 & 7.12 & 10.88 & 363 \\
$\mathrm{C} 2$ & 3.77 & 8.13 & 7.39 & 11.59 & 387 \\
\hline \hline
\end{tabular}
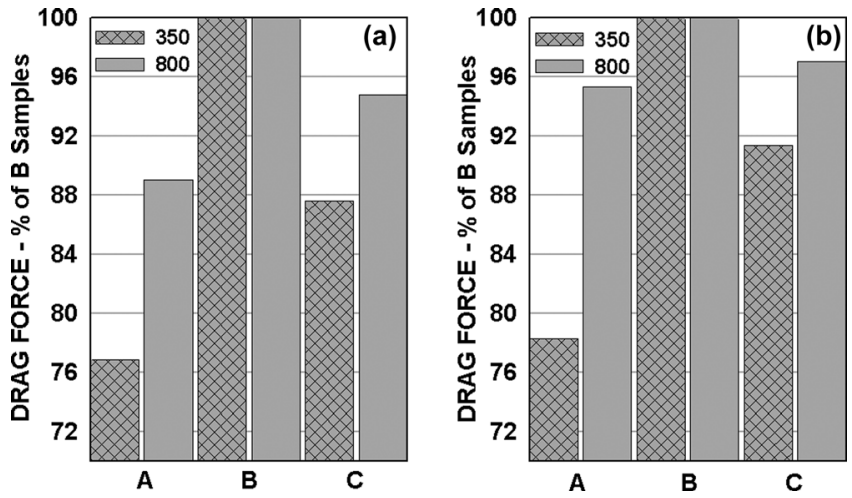

FIG. 3. Variations in $\Delta F$ when PM edge is coincident with SUT edge: (a) $6.35 \mathrm{~mm}$ wide PM; (b) $12.7 \mathrm{~mm}$ wide PM.

Two samples of each material and each cutting process (12 samples total) were prepared.

$\Delta F$ for each sample was measured in previously described apparatus ${ }^{5}$ using a $6.35 \mathrm{~mm}, 12.7 \mathrm{~mm}$, or $50.8 \mathrm{~mm}$ wide $\mathrm{NdFeB}, 42 \mathrm{MGOe}$ magnet, each being $6.35 \mathrm{~mm}$ high, $3.18 \mathrm{~mm}$ thick and polarized through its thickness. The 6.35 $\mathrm{mm}$ wide magnet was mounted in an aluminum holder in a manner allowing for adjustment of its position relative to the edge of the SUT ( $E$ in Fig. 1).

One end of the SUT was clamped to the moving portion of the apparatus. The other end was supported in a manner assuring that the edge being examined was parallel to the motion with both $G(=0.3 \mathrm{~mm})$ and $E$ held constant. The forward and back travel (STROKE in Fig. 2) was set to 66 $\mathrm{mm}$ at a speed slightly more than $2 \mathrm{~mm} / \mathrm{s}$. A linear encoder monitored the position of the SUT relative to the PM. The entire apparatus was tilted to apply a bias force large enough to keep the load cell in compression during motion in both directions. Force and position measurements were acquired by computer and by means of MATLAB ${ }^{\circledR}$ software, an average value of $\Delta F$ was determined for the central $26 \mathrm{~mm}$ portion of the STROKE (DATA in Fig. 2).

Measured values of $\Delta F$ for each sample, taken with the PMs indicated, are listed in Tables I and II. For the columns indicated as "Edge," $E=0$; for the columns indicated as "Center," the PM center was $15 \mathrm{~mm}$ from the guiding edge of the SUT. Hysteresis losses associated with the $50.8 \mathrm{~mm}$ data [integrated term in Eq. (1)] $=\Delta F / 2 A$, calculated from $\Delta F, W_{\mathrm{s}}$, and $t_{\mathrm{s}}$ measurements, are shown in the last column of each table. Since induction extrema under these measurement conditions are not known, nor are conventionally

TABLE II. $\Delta F$ for PMs and SUTs indicated ( 800 grade).

\begin{tabular}{lccccc}
\hline \hline $\mathrm{PM} \Rightarrow$ \\
$\mathrm{SUT} \Downarrow$ & $\begin{array}{c}6.35 \mathrm{~mm} \\
\text { Edge }(\mathrm{mN})\end{array}$ & $\begin{array}{c}12.7 \mathrm{~mm} \\
\text { Edge }(\mathrm{mN})\end{array}$ & $\begin{array}{c}12.7 \mathrm{~mm} \\
\text { Center }(\mathrm{mN})\end{array}$ & $\begin{array}{c}50.8 \mathrm{~mm} \\
\text { Center }(\mathrm{mN})\end{array}$ & $\begin{array}{c}\text { Hysteresis } \\
\text { loss }\left(\mathrm{J} / \mathrm{m}^{3}\right)\end{array}$ \\
\hline $\mathrm{A} 1$ & 6.22 & 15.03 & 13.86 & 25.28 & 818 \\
$\mathrm{~A} 2$ & 5.89 & 14.88 & 13.53 & 25.50 & 826 \\
$\mathrm{~B} 1$ & 6.74 & 15.53 & 14.24 & 25.75 & 839 \\
$\mathrm{~B} 2$ & 6.87 & 15.86 & 14.81 & 26.23 & 856 \\
$\mathrm{C} 1$ & 6.37 & 15.29 & 14.01 & 24.90 & 815 \\
$\mathrm{C} 2$ & 6.53 & 15.15 & 14.27 & 25.54 & 835 \\
\hline \hline
\end{tabular}



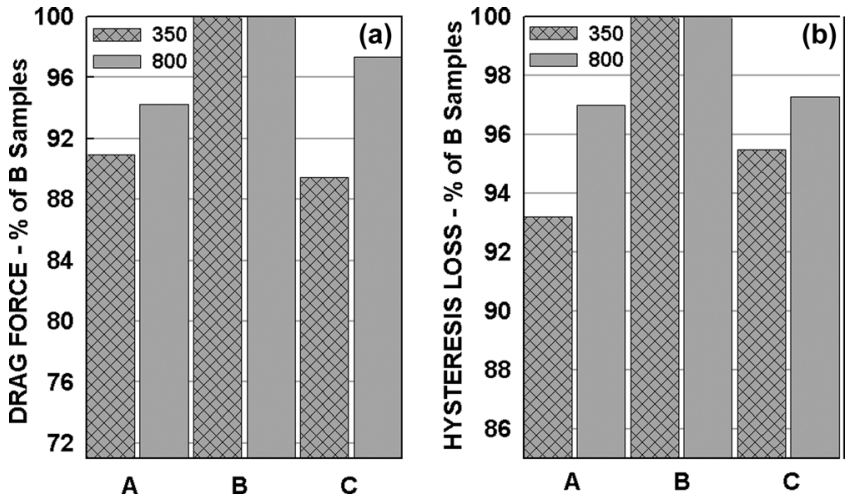

FIG. 4. (a) Effect of cutting process on $\Delta F$ measured in center of SUT with $12.7 \mathrm{~mm}$ PM. (b) Effect of cutting process on global hysteresis loss determined by drag force.

measured losses represented by the area of the "minor" loop having extrema at saturation and remanence (included in the DFM), the listed values cannot be critically compared with known values for these materials. Nevertheless, they illustrate how relative losses are derived from drag force measurements.

The $\Delta F$ "Edge" values from all B samples, with both PMs, are seen to be consistently largest, with those from the A samples, consistently smallest. This ranking is made even more apparent when comparing the averaged data from the two samples of each type in the graphical form shown in Figs. 3 and 4. The notably larger cutting process dependence exhibited by the 350 grade material is consistent with its expected higher stress sensitivity reflective of the larger magnetostriction $\left(\lambda_{\mathrm{s}}\right)$ and smaller crystal anisotropy $\left(K_{1}\right)$ associated with its higher silicon content. ${ }^{6}$

While the dependence of $\Delta F$ on PM location ( $E$ in Fig. 1) is as expected, its variation with PM width $\left(W_{\mathrm{M}}\right)$ requires further exploration. Figure 5 shows how the peak longitudinal components of fields from the three sizes of PM vary with position along their widths. We can clearly observe that the $50.8 \mathrm{~mm}$ PM will expectedly instill uniform magnetization across the full $30 \mathrm{~mm}$ wide samples. In contrast, the fields from the 12.7 and $6.35 \mathrm{~mm}$ PMs drop to $\sim 56 \%$ of their centerline intensities at their edges. If the field intensity at a PM edge is still intense enough to contribute to the observed $\Delta F$ then it is quite likely that the width of the magnetized band will be even (at least slightly) wider than $W_{\mathrm{M}}$. When $E=0$, such a band can extend only inward from

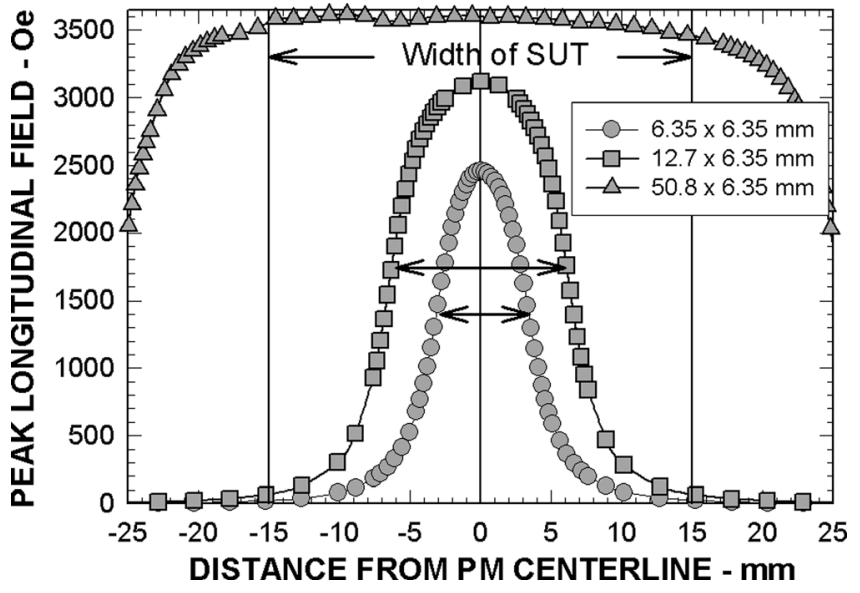

FIG. 5. Variation in longitudinal field components across the widths (indicated by arrows) of the indicated PMs. Hall effect element at $1 \mathrm{~mm}$ from PM's surface at the center of its thickness.

the edge, whereas when $E=\left(30-W_{\mathrm{M}}\right) / 2$, the band boundaries are limited only by the field intensity and the magnetic softness of the SUT. The average $\Delta F$ developed with the $12.7 \mathrm{~mm} \mathrm{PM}$ is $\sim 55 \%$ (800 grade SUTs, $\sim 66 \%$ for the 350 grade SUTs) of that developed with the $50.8 \mathrm{~mm}$ wide PM, and it is known that the full width data include contributions from regions where the hysteresis loss is higher than in the center (i.e., the edge regions). Thus, it can be concluded that the band magnetized by the $12.7 \mathrm{~mm}$ PM is wider than 12.7 $\mathrm{mm}$. Since the drag force data with this PM at an edge are almost always larger still than when it is at the center of the SUT, it can be further concluded that the larger hysteresis losses in the edge regions more than makeup for the lesser available width of magnetizable material. Details concerning the loss profile of the edge regions can expectedly be inferred from drag force measurements with the PM at varying distances from an edge. In any case, the utility of the drag force method for simply, quickly, and nondestructively assessing the intensity and extent of the degradation wrought by a cutting process seems well established.

\footnotetext{
${ }^{1}$ M. Emura et al., J. Magn. Magn. Mater., 254, 358 (2003).

${ }^{2}$ A. J. Moses et al., J. Magn. Magn. Mater. 215, 690 (2000).

${ }^{3}$ G. Crevecoeur et al., IEEE Trans. Magn. 44, 3173 (2008).

${ }^{4}$ G. Crevecoeur et al., IEEE Trans. Magn. 44, 1010 (2008).

${ }^{5}$ I. J. Garshelis et al., J. Appl. Phys. 99, 08D910 (2006).

${ }^{6}$ S. Chikazumi, Physics of Magnetism (Krieger, Malabar, FL, 1964), p. 145 and 174.
} 